\title{
Definitive Intensity-modulated Radiation Therapy in Elderly Patients with Locally Advanced Oropharyngeal Cancer
}

\author{
FRANCESCA DE FELICE ${ }^{1}$, ALESSANDRO GALDIERI ${ }^{1}$, GESSICA ABATE ${ }^{1}$, \\ NADIA BULZONETTI ${ }^{1}$, DANIELA MUSIO ${ }^{1}$ and VINCENZO TOMBOLINI ${ }^{1,2}$ \\ ${ }^{1}$ Department of Radiotherapy, Policlinico Umberto I, Sapienza University of Rome, Rome, Italy; \\ ${ }^{2}$ Spencer-Lorillard Foundation, Rome, Italy
}

\begin{abstract}
Background: To evaluate the treatment tolerance and clinical outcomes in patients aged 70 years and older with locally advanced oropharyngeal cancer treated by definitive intensity-modulated radiation therapy (IMRT). Patients and Methods: We retrospectively analyzed 15 consecutive elderly patients, with histologically-proven squamous cell carcinoma of the oropharynx, staged T3-4 with or without involved lymph nodes at diagnosis, who received definitive sequential IMRT (70 Gy; 2 Gy/fraction). Adult Comorbidity Evaluation-27 (ACE-27) score was calculated and its influence on treatment tolerance and clinical outcomes was analyzed. Results: A total of 15 patients were included with a median age of 77 years (range $=70-88$ years). At baseline, 8 patients $(53.3 \%$ ) had an ACE-27 score of 1 , and the remainder $(n=7,46.7 \%)$ had a comorbidity index of 0 . All patients completed programmed IMRT treatment, without any reduction of total dose. Oral pain and mucositis were the most common acute side-effects, classified as grade 3 in 6 patients (40\%) only. Xerostomia was reported in 13 patients ( $86.7 \%)$, without severe manifestation. There was no hematological toxicity. ACE-27 score was not related to higher severe acute toxicity. No patients experienced grade 3 or more late toxicity. Five-year overall survival and disease-free survival rates were $63.6 \%$ (95\% confidence interval $=32.7-83.3 \%) \quad$ and $55 \% \quad(95 \%$ confidence interval $=24.4-77.6 \%)$, respectively. Comorbidity score did not influence survival outcomes, both overall survival $(p=0.46)$ and disease-free survival $(p=0.55)$. Conclusion: Treatment tolerance, as well as survival outcomes were good in elderly
\end{abstract}

This article is freely accessible online.

Correspondence to: Francesca De Felice, Department of Radiotherapy, Policlinico Umberto I "Sapienza" University of Rome, Viale Regina Elena 326, 00161 Rome, Italy. E-mail fradefelice@hotmail.it

Key Words: Head and neck cancer, elderly, radiotherapy, oropharynx, survival, recurrence, metastasis, outcome. oropharyngeal cancer patients treated with definitive sequential IMRT. Due to age and comorbidity, no dose or volume reduction for IMRT should be considered in this setting of patients. A prospective randomized trial with a large sample size should be conducted to confirm our results.

Management of patients with oropharyngeal cancer is complex and requires multidisciplinary team involvement, especially in those with locally advanced stage disease. Over the years, the use of radiation therapy (RT) as primary treatment has markedly increased and the intensity-modulated technique (IMRT) is now the predominant application of RT used, due to its ability in optimizing dose distributions (1). Based on a large number of clinical trials showing significant increase in overall survival (OS), concomitant chemoradiotherapy (CRT) has become the standard treatment for locoregionally advanced oropharyngeal carcinoma (2). But CRT is still not considered as a standard of care for elderly patients. In fact, the vast majority of these randomized studies have not enrolled old ( $\geq 70$ years) patients and the updated meta-analysis of chemotherapy in head and neck cancer (MACH-NC) did not show any survival benefit resulting from the addition of chemotherapy for elderly patients, suggesting that the real impact of CRT remains to be determined in this setting for these patients (2).

Therefore, we felt that a more detailed look at elderly patients could be of interest. We tested definitive sequential IMRT in elderly patients with locally advanced oropharyngeal cancer. The aim of this study was to report the long-term follow-up data. The acute and late adverse effects were also determined.

\section{Patients and Methods}

Patient population. We retrospectively analyzed data from all consecutive elderly patients with locally advanced oropharyngeal cancer treated by definitive RT from January 2009 to August 2014. The study was approved by the Institutional Reviewed Board (October 10 2016, P-443/2016) and patients singed an informed consent form. Selection criteria for analysis cases included the 
following patients: the elderly ( $\geq 70$ years); those with newly diagnosed histologically proven squamous cell carcinoma of the orpharynx; those with clinical T3-4 disease, with or without involved lymph nodes and without distant metastasis at diagnosis; those without history of previous RT or chemotherapy. Patients' performance status was assessed by the Eastern Cooperative Oncology Group Performance Status (PS) score (3). The Adult Comorbidity Evaluation-27 (ACE-27) score, a 27-item validated comorbidity index specific for head and neck cancer, was used to analyze patient comorbidities (4).

Radiation therapy. RT was delivered using a standard fractionation at $2 \mathrm{~Gy} /$ fraction/day, 5 days/week, to a total dose of $70 \mathrm{~Gy} / 35$ fractions/7 weeks. Sequential IMRT technique was utilized. A total dose of $50 \mathrm{~Gy}$ was delivered to the low-risk target volume (sites of suspected subclinical spread), followed by the intermediate dose boost $(60 \mathrm{~Gy})$ to the entire anatomical subsite and the involved lymph node levels and highdose boost (70 Gy) to the primary tumor and pathological lymph nodes.

Follow-up. During RT, patients were examined daily. Once treatment ended, patients were evaluated at 6-week intervals for the first year, then every 3 months for the next 2 years, and every 6 months thereafter. Toxicity was evaluated using National Cancer Institute's Common Terminology Criteria for Adverse Events version 4.0 (5). Patients were followed-up closely to evaluate toxicities and detect persistent or recurrent disease by clinical and fiberoptic examinations; where appropriate computed tomography or magnetic resonance imaging was performed.

Statistical analysis. Statistical analysis was performed using RStudio-0.98.1091 software. Standard descriptive statistics were used to evaluate the distribution of each factor. Continuous data were given as median (range), and categorical data as the number of observations and ratios. Overall (OS) and disease-free (DFS) survival were calculated in months from the date of the end of RT to the date of the last follow-up, death (OS) and/or relapse (DFS).

Outcomes were estimated according to the Kaplan-Meier method and survival curves were compared using the log-rank test (6). In order to determine the influence on survival outcomes the variable ACE-27 was considered. Toxicity and compliance with treatment rates were also examined. The non-parametric Bernard test was used. Statistical tests were two-sided and $p$-values lower than 0.05 were considered significant.

\section{Results}

Patient characteristics. A total of 15 elderly patients were eligible for this analysis. Patient and tumor characteristics are listed in Table I. Ages ranged from 70 to 88 years, with a median of 77 years. At baseline, all patients had a PS score of 0, whereas eight patients $(53.3 \%)$ had an ACE-27 score of 1 , and the remainder $(n=7,46.7 \%)$ had a comorbidity index of 0 . The vast majority of patients $(n=13 ; 86.7 \%)$ had more than one positive regional lymph node at diagnosis.

Treatment compliance and toxicity. All patients received the prescribed total dose of IMRT. Overall, RT was interrupted
Table I. Patient and tumor characteristics.

\begin{tabular}{|c|c|}
\hline Characteristic & Value \\
\hline Median age (range), years & $77(70-88)$ \\
\hline \multicolumn{2}{|l|}{ Gender, n $(\%)$} \\
\hline Male & $9(60)$ \\
\hline Female & $6(40)$ \\
\hline \multicolumn{2}{|l|}{ PS, n (\%) } \\
\hline 0 & $15(100)$ \\
\hline 1 & $0(0)$ \\
\hline \multicolumn{2}{|l|}{ ACE-27 score, n (\%) } \\
\hline 0 & $7(46.7)$ \\
\hline 1 & $8(53.3)$ \\
\hline \multicolumn{2}{|l|}{ Smoker, n (\%) } \\
\hline No & $5(33.3)$ \\
\hline Current & $7(43.7)$ \\
\hline Former & $3(20)$ \\
\hline \multicolumn{2}{|l|}{ Primary tumor site, $\mathrm{n}(\%)$} \\
\hline Tonsil & $11(73.3)$ \\
\hline Soft palate & $1(6.7)$ \\
\hline Base of tongue & $3(20)$ \\
\hline \multicolumn{2}{|l|}{ Clinical tumor stage, n (\%) } \\
\hline $\mathrm{T} 1-2$ & $9(60)$ \\
\hline T3-4 & $6(40)$ \\
\hline \multicolumn{2}{|l|}{ Clinical nodal stage, n (\%) } \\
\hline N0-1 & $2(13.3)$ \\
\hline $\mathrm{N} 2-3$ & $13(86.7)$ \\
\hline
\end{tabular}

for a mean period of 5 days for acute toxicity in two patients $(13.3 \%)$.

All patients had acute toxicity associated with treatment. Details are shown in Table II. Mucositis and oral pain were the most common acute symptoms. In total, the incidence rate of any severe acute toxicity was $37.5 \%(n=9)$. Xerostomia was reported in 13 patients $(86.7 \%)$, without severe manifestation. Six patients $(40 \%)$ had tube feeding due to inadequate oral caloric intake. There were no life-threatening consequences. No significant difference in higher severe acute toxicity was found regarding ACE-27 score (0 versus $\geq 1$ ).

Late RT treatment-related toxicity was assessed among surviving patients and involved the following conditions: xerostomia $(n=9)$, oral pain $(n=1)$, dysgeusia $(n=5)$, trismus $(n=1)$, dysphagia $(n=5)$, brachial plexus injury $(n=1)$. There was no evidence of osteoradionecrosis, nor of ototoxicity. None experienced grade 3 or more late toxicity.

Clinical outcomes. Overall, six patients deaths were recorded, two $(33.3 \%)$ from conditions unrelated to cancer. The 2- and 5-year OS rates were $72.7 \%(95 \% \mathrm{CI}=42.5-88.8 \%)$ and $63.6 \%(95 \% \mathrm{CI}=32.7-83.3 \%)$, respectively (Figure 1). Locoregional recurrence was recorded in 2 patients $(13.3 \%)$ and only one patient $(6.7 \%)$ presented distant metastasis to lung. Two- and 5- year DFS were estimated at 66\% $(95 \%$ $\mathrm{CI}=36.5-84.3 \%)$ and $55 \%(95 \% \mathrm{CI}=24.4-77.6 \%)$, respectively 


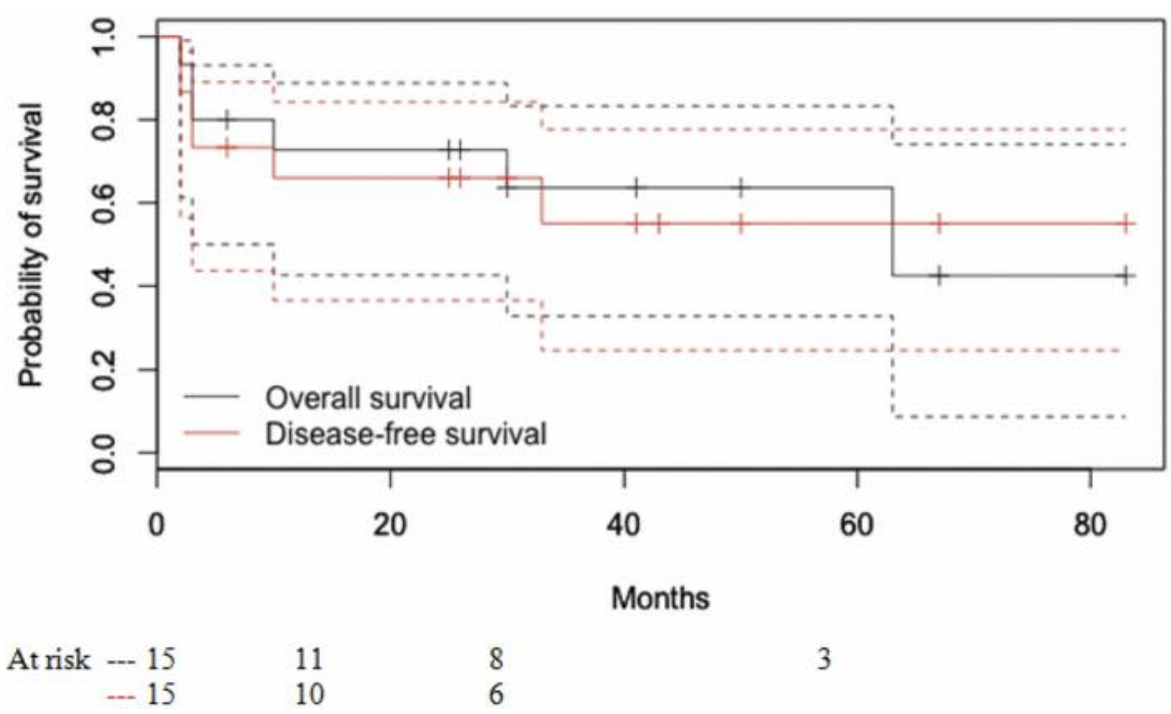

Figure 1. Kaplan-Meier curves of overall survival and disease-free survival of elderly patients after intensity-modulated radiation therapy.

Table II. Acute toxicity experienced by elderly patients after intensity-modulated radiation therapy.

\begin{tabular}{|c|c|c|c|c|c|c|c|c|}
\hline \multirow[b]{3}{*}{ Toxicity } & \multicolumn{3}{|c|}{ Grade $1-2$} & \multirow[b]{3}{*}{$p$-Value } & \multicolumn{3}{|c|}{ Grade $3-4$} & \multirow[b]{3}{*}{$p$-Value } \\
\hline & \multicolumn{3}{|c|}{ ACE-27 score, n (\%) } & & \multicolumn{3}{|c|}{ ACE-27 score, n (\%) } & \\
\hline & All (\%) & $0(\%)$ & $1(\%)$ & & All (\%) & $0(\%)$ & $1(\%)$ & \\
\hline Dermatitis radiation & $1(6.7)$ & $1(14.3)$ & - & 0.63 & $1(6.7)$ & - & $1(12.5)$ & 0.73 \\
\hline Mucositis oral & $11(73.3)$ & $4(57.1)$ & $7(87.5)$ & 0.66 & $4(26.7)$ & $3(42.9)$ & $1(12.5)$ & 0.42 \\
\hline Odinophagia/oral pain & $13(86.7)$ & $6(85.7)$ & $7(87.5)$ & 0.97 & $2(13.3)$ & $1(14.3)$ & $1(12.5)$ & 0.97 \\
\hline Xerostomia & $13(86.7)$ & $5(71.4)$ & $8(100)$ & 0.76 & - & - & - & \\
\hline Dysgeusia & $13(86.7)$ & $5(71.4)$ & $8(100)$ & 0.76 & - & - & - & \\
\hline Dysphagia & $6(46.2)$ & $3(42.9)$ & $3(37.5)$ & 0.96 & $2(13.3)$ & $2(28.6)$ & - & 0.72 \\
\hline Dysphonia & $3(20)$ & $3(42.9)$ & $1(12.5)$ & 0.42 & - & - & - & \\
\hline Neutrophil count decreased & $1(6.7)$ & - & $1(12.5)$ & 0.73 & - & - & - & \\
\hline
\end{tabular}

ACE-27: Adult comorbidity index-2.

(Figure 1). ACE-27 score had no influence on survival outcomes. A 75\% (95\% CI=31.5-93.1\%) 2-year survival was recorded in patients with ACE-27 score 0 and $71.4 \%(95 \%$ $\mathrm{CI}=25.8-0.92)$ in patients with ACE-27 score $1(p=0.46)$. In 2-year disease free survival probabilities comparable results were found ( $75 \%$ versus $57.1 \%$, respectively; $p=0.55$ )

\section{Discussion}

This study provided a reliable evaluation of the effect of sequential IMRT in elderly patients with locally advanced oropharyngeal cancer. Results suggested that definitive IMRT with standard fractionation was effective in reducing mortality and both local and distant recurrences, without deleterious effect on compliance and toxicity. The ACE-27 score was not related to survival and toxicity rates.

Although approximately $25 \%$ of patients with head and neck cancer are aged 70 years and over, over the past decade elderly patients have represented fewer than $10 \%$ of clinical trial participants $(7,8)$. This under-representation of the elderly suggests that it is necessary to better understand agebased differences in presentation, response to therapy and prognosis. Further studies should be designed exclusively for elderly patients. It is expected that they have similar comorbidities that could affect compliance with or response to treatment. Evaluation and quantification of comorbidity is paramount to stratifying elderly patients into groups with similar pre-treatment health condition that impacts on short- 
term mortality and the ACE-27 index is an established tool in head and neck squamous cell carcinoma assessment $(8,9)$. Interestingly, our results showed no significant differences in clinical outcomes, toxicity manifestation and compliance with IMRT. This favorable profile could be explained by the good performance of the enrolled patients. In fact, a clear distinct impact of the ACE-27 severity scores was shower here. Our results are in agreement with survival rates found in the study of Datema et al., in which the 2-year survival probability of a patient with ACE-27 score 1 was $67 \%$ against $75 \%$ for a patient with ACE-27 score 0 (9). To our knowledge, in the head and neck cancer scenario, there is only one published prospective study that required an age from 65 to 79 years (10). However, this study was performed to assess the efficacy and safety profiles of the induction chemotherapy, and did not contain enough RT data to allow a proper comparison of results. Based on five trials of the European Organization for Research and Treatment of Cancer restricted to patients receiving RT for head and neck cancer, Pignon et al. analyzed locoregional control, survival and side-effects in relation to the age of the patients (11). Globally, 1,589 patients were enrolled and 185 patients were over 70 years $(11.6 \%)$. Although under-represented, the absolute number of elderly patients was considered sufficient to perform the analysis. Overall, acute RT tolerance was worse in elderly patients than younger patients, whereas there were no differences in term of local control and OS between age groups. The authors concluded that an age limit was not a good strategy to guide administration of head and neck cancer therapy. However, there are two main considerations. Firstly, their study had no information regarding comorbidity. Secondly, patient data were from 1980 to 1995 and tolerance rates may have been negatively influenced by RT technique. Recently, Nguyen et al. presented a series of 27 patients with locally advanced head and neck cancer aged $\geq 70$ years treated with concurrent CRT (12). Only 11 cases had primary oropharyngeal neoplasia but no subgroup analysis for anatomic sites was available. The 2 -year OS of the entire elderly cohort was $67.5 \%$. In total, 16 patients developed grade 3 or more acute mucositis, seven had grade 3 or more hematological toxicity, five had severe skin reaction and three presented severe vomiting. The mean treatment break was 7 days. Our patients experienced a less severe toxicity profile and we believe this was because IMRT was not combined with chemotherapy. Moreover, if we compare results, our survival rate was better, but due to the short follow-up (median 12 months) observed by Nguyen et al., no definitive conclusions can be drawn.

Our study could be useful to identify the optimal therapeutic strategy in elderly patients with locally advanced oropharyngeal cancer. The use of sequential IMRT appears to be safe and efficacious. Our employed RT fractionation schedule is justified by radiobiological research and clinical data. It has been well demonstrated that hyperfractionation with dose escalation results in consistent survival benefit with an increase of severe acute side-effects, especially mucosal toxicity, compared with conventional fractionation (13-15). On the other hand, shortening the overall treatment time using accelerated fractionation improves locoregional control, but acute and late toxicities are also increased (16). Moreover, a recent metaanalysis showed an absolute survival benefit for altered fractionation of $3.4 \%$ at 5 years, but this benefit was limited to patients younger than 60 years of age (17). Therefore, considering that a consensus regarding hyperfractionation and altered fraction schedules has not yet emerged, and considering patient age and comorbidity, the higher toxicity risk outweighed the survival advantage and thus the conventional fractionation was chosen. In addition, the application of the IMRT technique was useful in reducing late toxicity, by sparing closely adjacent critical anatomic structures, such as salivary glands, pharyngeal constrictor muscles, mandible, temporomandibular joint and masticatory muscles.

However, our results should be viewed with caution. The retrospective nature of this analysis is the main limitation of the study. The sample size was relatively small, making results less robust. But homogeneity in the patient population and in the treatment approach, without missing data, represent the study strengths. Considering the lack of literature evidence on treatment recommendations for older patients with locally advanced oropharyngeal cancer, this study could be useful to assist the radiation oncologist in elderly patient counseling and clinical decision-making.

\section{Conclusion}

No general consensus exists for appropriate treatment in elderly patients with oropharyngeal cancer. A high degree of locoregional and distant control can be achieved with definitive sequential IMRT, with high compliance and tolerable toxicity. The definitive sequential IMRT technique should be considered a valid option in this patient setting. Further studies are paramount to confirm that elderly patients can benefit from this treatment approach.

\section{References}

1 National Comprehensive Cancer Network Guidelines Head and Neck Version 1.2016 https://www.nccn.org/professionals/ physician_gls/pdf/head-and-neck.pdf

2 Pignon JP, le Maître A, Maillard E, Bourhis J; MACH-NC Collaborative Group: Meta-analysis of chemotherapy in head and neck cancer (MACH-NC): an update on 93 randomised trials and 17,346 patients. Radiother Oncol 92(1): 4-14, 2009.

3 Oken MM, Creech RH, Tormey DC, Horton J, Davis TE, McFadden ET and Carbone PP: Toxicity and response criteria of the Eastern Cooperative Oncology Group. Am J Clin Oncol 5(6): 649-655, 1982. 
4 Piccirillo JF, Tierney RM, Costas I, Grove L and Spitznagel EL Jr.: Prognostic importance of comorbidity in a hospital-based cancer registry. JAMA 291(20): 2441-2447, 2004.

5 Cancer Therapy Evaluation Program (2009) Common Terminology Criteria for Adverse Events, Version 4.0. Available from: URL: http://ctep.cancer.gov

6 Schemper M and Smith TL: A note on quantifying follow-up in studies of failure time. Control Clin Trials 17(4): 343-346, 1996.

7 Murthy VH, Krumholz HM and Gross CP: Participation in cancer clinical trials: race-, sex-, and age-based disparities. JAMA 291(22): 2720-2726, 2004.

8 Hartmann S and Grandis JR: Treatment of head and neck cancer in the elderly. Expert Opin Pharmacother 17(14): 1903-1921, 2016.

9 Datema FR, Ferrier MB, van der Schroeff MP and Baatenburg de Jong RJ: Impact of comorbidity on short-term mortality and overall survival of head and neck cancer patients. Head Neck 32(6): 728-736, 2010.

10 Choi YJ, Chung J, Shin HJ, Cho GJ, Wang SG, Lee BJ, Cho BM, Kim DW, Kim HJ, Lee WS, Joo YD and Sohn CH: Induction chemotherapy of docetaxel and Cisplatin for the elderly patients with squamous cell carcinoma of the head and neck. Cancer Res Treat 39(1): 1-5, 2007.

11 Pignon T, Horiot JC, Van den Bogaert W, Van Glabbeke M and Scalliet P: No age limit for radical radiotherapy in head and neck tumours. Eur J Cancer 32A(12): 2075- 2081,1996.

12 Nguyen NP, Vock J, Chi A, Vinh-Hung V and Dutta S, Ewell L, Jang S, Betz M, Almeida F, Miller M, Davis R, Sroka T, Vo RP, Karlsson U and Vos P: Impact of intensity-modulated and imageguided radiotherapy on elderly patients undergoing chemoradiation for locally advanced head and neck cancer. Strahlenther Onkol 188(8): 677-683, 2012.

13 Budach W, Hehr T, Budach V, Belka C and Dietz K: A metaanalysis of hyperfractionated and accelerated radiotherapy and combined chemotherapy and radiotherapy regimens in unresected locally advanced squamous cell carcinoma of the head and neck. BMC Cancer 6: 28, 2006.
14 Horiot JC, Le Fur R, N'Guyen T, Chenal C, Schraub S, Alfonsi S, Gardani G, Van Den Bogaert W, Danczak S and Bolla M: Hyperfractionation versus conventional fractionation in oropharyngeal carcinoma: final analysis of a randomized trial of the EORTC cooperative group of radiotherapy. Radiother Oncol 25(4): 231-241, 1992.

15 Konski AA, Winter K, Cole BF, Ang KK and Fu KK: Qualityadjusted survival analysis of Radiation Therapy Oncology Group (RTOG) 90-03: phase III randomized study comparing altered fractionation to standard fractionation radiotherapy for locally advanced head and neck squamous cell carcinoma. Head Neck 31(2): 207-212, 2009.

16 Horiot JC, Bontemps P, van den Bogaert W, Le Fur R, van den Weijngaert D, Bolla M, Bernier J, Lusinchi A, Stuschke M, LopezTorrecilla $\mathrm{J}$ and Begg AC, Pierart M and Collette L: Accelerated fractionation (AF) compared to conventional fractionation $(\mathrm{CF})$ improves loco-regional control in the radiotherapy of advanced head and neck cancers: results of the EORTC 22851 randomized trial. Radiother Oncol 44(2): 111-121, 1997.

17 Bourhis J, Overgaard J, Audry H, Ang KK, Saunders M, Bernier J, Horiot JC, Le Maître A, Pajak TF, Poulsen MG, O'Sullivan B, Dobrowsky W, Hliniak A, Skladowski K, Hay JH, Pinto LH, Fallai C, Fu KK, Sylvester R, Pignon JP; Meta-Analysis of Radiotherapy in Carcinomas of Head and neck (MARCH) Collaborative Group. Hyperfractionated or accelerated radiotherapy in head and neck cancer: a meta-analysis. Lancet 368(9538): 843-854, 2006.
Received January 28, 2017

Revised March 11, 2017

Accepted March 14, 2017 